\title{
ON SUBORDINATED HOLOMORPHIC SEMIGROUPS
}

\author{
ALFRED S. CARASSO AND TOSIO KATO
}

\begin{abstract}
If $\left[e^{-t A}\right]$ is a uniformly bounded $C_{0}$ semigroup on a complex Banach space $X$, then $-A^{\alpha}, 0<\alpha<1$, generates a holomorphic semigroup on $X$, and $\left[e^{-t A^{\alpha}}\right]$ is subordinated to $\left[e^{-t A}\right]$ through the Lévy stable density function. This was proved by Yosida in 1960, by suitably deforming the contour in an inverse Laplace transform representation. Using other methods, we exhibit a large class of probability measures such that the subordinated semigroups are always holomorphic, and obtain a necessary condition on the measure's Laplace transform for that to be the case. We then construct probability measures that do not have this property.
\end{abstract}

\section{INTRODUCTION}

Let $X$ be a complex Banach space, and let $C_{0}(X)$ be the class of uniformly bounded $C_{0}$ semigroups $[T(t)], t \geq 0$, on $X$. For fixed $\alpha, 0<\alpha<1$, let $\left[p_{u}^{\alpha}(t)\right]$ be the family of functions implicitly defined as follows in Laplace transform space:

$$
\mathscr{L}\left\{p_{u}^{\alpha}(t)\right\} \equiv \int_{0}^{\infty} p_{u}^{\alpha}(t) e^{-u z} d u=e^{-t z^{\alpha}}, \quad \operatorname{Re} z>0 .
$$

The principal branch of $z^{\alpha}$ is understood in (1). For each fixed $t>0, p_{u}^{\alpha}(t)$ is a Lévy 'stable' probability density function on $u \geq 0$. Given $[T(u)] \in C_{0}(X)$, one may use (1) to construct a new semigroup $[U(t)] \in C_{0}(X)$, by means of

$$
U(0)=I, \quad U(t) x=\int_{0}^{\infty} p_{u}^{\alpha}(t) T(u) x d u, \quad t>0, x \in X .
$$

We express this symbolically by $U(t)=\left\langle p^{\alpha}(t), T\right\rangle$, where, for fixed $t, p^{\alpha}(t)$ is the probability distribution with density $p_{u}^{\alpha}(t)$. We write $T(t)=e^{-t A}$, where $-A$ is the infinitesimal generator of $[T(t)]$. Whenever multivalued functions $\psi(z)$ appear, the particular branch where $\operatorname{Re} \psi(z)>0$ for $\operatorname{Re} z>0$, is understood.

The above is an example of a subordinated semigroup: $[U(t)]$ is said to be subordinated to $[T(t)]$ through the directing process $\left[p^{\alpha}(t)\right]$. See e.g. Feller,

Received by the editors September 12, 1989.

1980 Mathematics Subject Classification (1985 Revision). Primary 47D05, 60E07, 60J25.

Key words and phrases. Subordinated semigroups, holomorphic extensions.

The first author's work was supported in part by the Office of Naval Research under contract number N00014-89-F-0013; the second by NSF Grant DMS-8701724. 
[5, pp. 345-349]. The concept originated with Bochner, [3, 4], who used (1) and (2) to construct $A^{\alpha}, 0<\alpha<1$. Subsequently, Phillips, [10], Nelson, [7], and Balakrishnan, [1], considered arbitrary infinitely divisible probability distributions on $u \geq 0$, and developed a functional calculus for semigroup generators. Alternative methods of constructing fractional powers of operators, independent of subordination, were later devised by several authors, spawning a large literature; see Pazy, [9, p. 257]. Returning to (2), Yosida, [13-15], drew attention to the fact that in that case the semigroup $[U(t)]$ is holomorphic, and that (1) and (2) together provide a method of constructing a large subclass of holomorphic semigroups within the class $C_{0}$. However, no other examples of families $[p(t)]$ leading to subordinated holomorphic semigroups seem generally known.

In this paper, we exhibit a rich variety of semigroups $[p(t)]$ of probability measures, such that $[U(t)]=[\langle p(t), T\rangle]$ is holomorphic whenever $[T(t)] \in$ $C_{0}(X)$, and we obtain a necessary condition on $\mathscr{L}\{p(t)\}$ in order that this be the case. We also construct families $[p(t)]$ that do not have this property.

\section{SEMIGROUPS OF PROBABILITY MEASURES}

This section summarizes known results; see Phillips, [10], Hille and Phillips, [6, pp. 660-663], and Feller, [5]. Let $B(X)$ be the Banach algebra of bounded linear operators on $X$. Let $S$ be the Banach algebra of complex Borel measures $\mu$ on $\mathbf{R}^{+} \equiv\{u \geq 0\}$, with convolution as multiplication, and normed by the total variation. If $V$ is a Borel set $\subset \mathbf{R}^{+}, \mu(V)$ denotes the value of $\mu$ on $V$, while $\int_{V} g(u) \mu(d u)$ is the integral with respect to $\mu$ of the Borel measurable function $g$. Let $L$ be the Banach space of Borel measurable functions $f$ on $\mathbf{R}^{+}$such that

$$
\|f\|_{L}=\int_{\mathbf{R}^{+}}|f(u)| d u<\infty .
$$

For each $\mu \in S$, define $Z_{\mu} \in B(L)$ by

$$
Z_{\mu} f=(\mu * f)(\tau) \equiv \int_{\mathbf{R}^{+}} f(\tau-u) \mu(d u), \quad f \in L, \tau \geq 0 .
$$

Then, the map $\mu \mapsto Z_{\mu}$ is an isometric isomorphism of $S$ into $B(L)$. Let $[T(u)] \in C_{0}(X)$. For each $\mu \in S$, define

$$
\langle\mu, T\rangle=\int_{\mathbf{R}^{+}} T(u) \mu(d u) .
$$

Then, $\mu \mapsto\langle\mu, T\rangle$, is a continuous homomorphism of $S$ into $B(X)$. In particular, $\langle\mu * \nu, T\rangle=\langle\mu, T\rangle\langle\nu, T\rangle$.

For each $x \geq 0$, let $\delta_{x}$ denote the Dirac measure at $x$, i.e., $\delta_{x}(V)=1$ if $x \in V, \delta_{x}(V)=0$ if $x \notin V$. Let $P$ be the set of all algebraic semigroups $[p(t)], t \geq 0$, of probability measures on $\mathbf{R}^{+}$. Thus, for fixed $t, p(t) \in S$, $p(t) \geq 0,\|p(t)\|_{S}=1, p(t) * p(s)=p(t+s), s, t \geq 0$, and $p(0)=\delta_{0}$. If $[p(t)] \in P$, then $\left[Z_{p}(t)\right] \equiv\left[Z_{p(t)}\right]$ forms an algebraic contraction semigroup 
on $L$, and for $[T(u)] \in C_{0}(X),[U(t)]=[\langle p(t), T\rangle]$ is a uniformly bounded algebraic semigroup on $X$. [U(t)] is subordinated to $[T(t)]$.

Definition 1. $\mathcal{I}$ is the set of all $[p(t)] \in P$ such that, given an arbitrary complex Banach space $X,[\langle p(t), T\rangle] \in C_{0}(X)$ whenever $[T(t)] \in C_{0}(X)$.

Theorem 1. Let $[p(t)] \in P$. The following statements are equivalent:

(a) $[p(t)] \in \mathscr{I}$.

(b) $\left[Z_{p}(t)\right] \in C_{0}(L)$.

(c) For every $x>0, p(t)\left(V_{x}\right) \rightarrow 1$ as $t \downarrow 0$, where $V_{x} \equiv\{0 \leq u \leq x\}$.

For fixed $t \geq 0$, define the Laplace transform of $p(t) \in S$ by

$$
\mathscr{L}\{p(t)\}=\int_{\mathbf{R}^{+}} e^{-u z} p(t)(d u), \quad \operatorname{Re} z>0 .
$$

Theorem 2. The following statements are equivalent:

(a) $[p(t)] \in \mathscr{I}$.

(b) $\mathscr{L}\{p(t)\}=e^{-t \psi(z)}, t \geq 0$, where $\psi(z)$ is holomorphic for $\operatorname{Re} z>0$ and continuous. for $\operatorname{Re} z \geq 0$, with $\operatorname{Re} \psi(z) \geq 0$. Moreover, $\psi(0)=0$, and $\psi^{\prime}(x)$ is completely monotone for $x>0$.

When $[p(t)] \in \mathscr{I}$, the function $\psi(z)$ is called the exponent of $[p(t)]$. An equivalent characterization of $\psi(z)$ is the following: There exists a positive measure $\rho$ on $\mathbf{R}^{+}$, finite or infinite, such that $\int_{u>1} u^{-1} \rho(d u)<\infty$, and

$$
\psi(z)=\int_{\mathbf{R}^{+}}\left(1-e^{-u z}\right) u^{-1} \rho(d u), \quad \operatorname{Re} z \geq 0 .
$$

A few objects $\in \mathscr{I}$ are known explicitly as functions of $u$ for all $t \geq 0$. In the following examples, $p_{u}(t)$ denotes the density of the probability distribution $p(t)$ on $\mathbf{R}^{+}$.

Degenerate.

$$
p(t)=\delta_{t}, \quad \mathscr{L}\{p(t)\}=e^{-t z}, \quad t>0 .
$$

Inverse Gaussian. This is the special case $\alpha=1 / 2$ in (1).

$$
p_{u}(t)=\frac{t e^{-t^{2} / 4 u}}{\sqrt{4 \pi u^{3}}}, \quad \mathscr{L}\{p(t)\}=e^{-t \sqrt{z}}, \quad t>0 .
$$

Gamma. With fixed $b>0$,

$$
p_{u}(t)=\frac{b^{t} u^{t-1} e^{-b u}}{\Gamma(t)}, \quad \mathscr{L}\{p(t)\}=b^{t}(z+b)^{-t}, \quad t>0 .
$$

Negative binomial. This is a discrete family consisting of a weighted sum of Dirac measures. With fixed $0<b<1$ and $a=1-b$,

$$
p(t)=b^{t} \sum_{j=0}^{\infty}\left(\begin{array}{c}
-t \\
j
\end{array}\right)(-a)^{j} \delta_{j}, \quad \mathscr{L}\{p(t)\}=b^{t}\left(1-a e^{-z}\right)^{-t}, \quad t>0
$$


Poisson. This is also a discrete family. With fixed $c>0$

$$
p(t)=e^{-c t} \sum_{j=0}^{\infty} \frac{(c t)^{j}}{j !} \delta_{j}, \quad \mathscr{L}\{p(t)\}=e^{c t\left(e^{-z}-1\right)}, \quad t>0 .
$$

Compound Poisson. Let $q$ be an arbitrary probability measure on $\mathbf{R}^{+}$, and let $Q(z)=\mathscr{L}\{q\}$. With $\{q\}^{* 0} \equiv \delta_{0}$ and fixed $c>0$,

$$
p(t)=e^{-c t} \sum_{j=0}^{\infty} \frac{(c t)^{j}}{j !}\{q\}^{* j}, \quad \mathscr{L}\{p(t)\}=e^{c t(Q(z)-1)}, \quad t>0 .
$$

This construction includes many explicitly known semigroups $\in \mathscr{I}$ as special cases. Thus, (12) corresponds to the choice $q=\delta_{1}$. Similarly, (11) is a special case of (13) with $c=-\log b>0$, and

$$
c Q(z)=-\log \left(1-a e^{-z}\right)=\sum_{j=1}^{\infty} \frac{a^{j} e^{-j z}}{j}, \quad \operatorname{Re} z \geq 0,
$$

so that $c q=\sum_{j=1}^{\infty} a^{j} \delta_{j} / j$. As another example, let $q$ have the density $q_{u}=$ $b e^{-b u}, b>0$. Then

$$
\left\{q_{u}\right\}^{* n}=\frac{b^{n} u^{n-1} e^{-b u}}{\Gamma(n)}, \quad n \geq 1,
$$

and $p(t)$ can be expressed in terms of the modified Bessel function $I_{1}$. With $c=1, p(t)=e^{-t} \delta_{0}+r(t)$, where $r(t)$ has the density

$$
r_{u}(t)=e^{-t}(b t / u)^{1 / 2} e^{-b u} I_{1}(2 \sqrt{b t u}), \quad t>0,
$$

and

$$
\mathscr{L}\{p(t)\}=e^{-t} e^{b t(z+b)^{-1}}, \quad t>0 .
$$

\section{HOLOMORPHIC SEMIGROUPS}

We consider bounded holomorphic semigroups $[S(t)]$ on $X$, for which $t$ can assume complex values in a sector

$$
\Sigma_{\omega}=\{t \in \mathbf{C}: \operatorname{Re} t>0,|\operatorname{Arg}(t)|<\omega\}, \quad 0<\omega \leq \pi / 2,
$$

with $\omega$ fixed. The family $[S(t)]$ is assumed to satisfy the following:

(a) $S(t)$ is a holomorphic function of $t \in \Sigma_{\omega}$.

(b) $S\left(t_{1}\right) S\left(t_{2}\right)=S\left(t_{1}+t_{2}\right), t_{1}, t_{2} \in \Sigma_{\omega}$.

(c) If $0<\varepsilon<\omega$, then $\|S(t)\|_{X} \leq M_{\varepsilon}<\infty$, for $t \in \Sigma_{\omega-\varepsilon}$.

(d) $S(0)=I$, and, within any sector $\Sigma_{\omega-\varepsilon}$ with $0<\varepsilon<\omega, S(t)$ is strongly continuous at $t=0$.

The following result, due to Yosida, [12], tells us when a given semigroup $[U(t)] \in C_{0}(X)$, defined on $t \geq 0$, can be extended to a bounded holomorphic semigroup $[S(t)]$ in some sector $\Sigma_{\omega}$. Note that (20) below together with $\|U(t)\|_{X} \leq M<\infty$, imply

$$
\sup _{t>0}\left\{t\left\|\left(e^{-\beta t} U(t)\right)^{\prime}\right\|_{X}\right\} \leq C_{\beta}<\infty,
$$

for any $\beta>0$. 
Theorem 3. Let $[U(t)], t \geq 0, \in C_{0}(X)$ with infinitesimal generator $-A$. Let $U(t) X \subset D(A)$ for all $t>0$, and let

$$
\limsup _{t \downarrow 0}\left\{t\|A U(t)\|_{X}\right\}<\infty .
$$

Then, for any $\beta>0,\left[e^{-\beta t} U(t)\right]$ can be extended to a bounded holomorphic semigroup $[S(t)]$ in some sector $\Sigma_{\omega}$.

\section{SUbORDinAtion AND THE ClASS $\mathscr{H}$}

Definition 2. For any complex Banach space $X, H(X) \subset C_{0}(X)$ is the class of semigroups on $X$ satisfying the hypotheses of Theorem $3 ; G(X) \subset H(X)$ is the class of semigroups with bounded generators; $\mathscr{H}[$ resp. $\mathscr{G}]$ is the set of all $[p(t)] \in \mathscr{I}$ such that for every $X,[\langle p(t), T\rangle] \in H(X)$ [resp. $G(X)]$ whenever $[T(t)] \in C_{0}(X)$.

We have $\mathscr{G} \subset \mathscr{H} \subset \mathcal{F}$. The degenerate family (8) is evidently $\notin \mathscr{H}$, while the inverse Gaussian (9), and all other one-sided Lévy families (1), belong to $\mathscr{H}$ as shown by Yosida, [13].

Theorem 4. Let $[p(t)] \in \mathscr{I}$. The following conditions are equivalent:

(a) $[p(t)] \in \mathscr{H}$.

(b) $\left[Z_{p}(t)\right] \in H(L)$.

(c) $p(t)$ is continuously differentiable $\in S$ for $t>0$, with $\left\|p^{\prime}(t)\right\|_{S}=O\left(t^{-1}\right)$ as $t \downarrow 0$.

Moreover, $[p(t)] \in \mathscr{H}$ only if $\psi(z)$ maps $\operatorname{Re} z>0$ into a truncated sector of opening $<\pi$, and there exist constants $K>0$, and $\gamma, 0<\gamma<1$, such that

$$
|\psi(z)| \leq K|z|^{\gamma}, \quad|z| \geq 1, \quad \operatorname{Re} z \geq 0 .
$$

Proof. (a) $\Rightarrow(\mathrm{b})$. Let $[p(t)] \in \mathscr{H}$. Using (4) and (5), $Z_{p}(t)=\langle p(t), T\rangle$, where $[T(u)]$ is the semigroup of right translations on $L$. Hence, $\left[Z_{p}(t)\right] \in H(L)$. (b) $\Rightarrow(\mathrm{c})$. Since $\left[Z_{p}(t)\right]$ satisfies the hypotheses of Theorem 3 on $L$, the $B(L)$ limit as $h \rightarrow 0$ of $h^{-1}\left\{Z_{p}(t+h)-Z_{p}(t)\right\}$ exists for each fixed $t>0$. By the isometric isomorphism $p(t) \mapsto Z_{p}(t), h^{-1}\{p(t+h)-p(t)\}$ has a corresponding $S$ limit $p^{\prime}(t)$, and $\left\|p^{\prime}(t)\right\|_{S}=\left\|Z_{p}^{\prime}(t)\right\|_{L}, t>0$. Therefore, from (20)

$$
\limsup _{t \downarrow 0}\left\{t\left\|p^{\prime}(t)\right\|_{S}\right\}<\infty .
$$

If $p^{\prime}(t) \in S$ for each $t>0$, the same is true of $p^{\prime}(t / 2) * p^{\prime}(t / 2)$. By considering $\mathscr{L}\{p(t)\}=e^{-t \psi(z)}$, it follows that $p^{\prime}(t / 2) * p^{\prime}(t / 2)=p^{\prime \prime}(t)$, so that $\left\|p^{\prime \prime}(t)\right\|_{S} \leq$ $\left\|p^{\prime}(t / 2)\right\|_{S}^{2}$. In particular, $\left\|p^{\prime}(t)\right\|_{S}$ is a bounded continuous function of $t$ on any interval $0<t_{0} \leq t \leq t_{1}<\infty$.

(c) $\Rightarrow\left(\right.$ a) Given any $[T(u)] \in C_{0}(X)$, differentiation with respect to $t$ under the integral sign is justified in $\langle p(t), T\rangle$. Hence, $U^{\prime}(t)=\left\langle p^{\prime}(t), T\right\rangle, t>0$, and

$$
\left\|U^{\prime}(t)\right\|_{X} \leq \text { const. }\left\|p^{\prime}(t)\right\|_{S}, \quad t>0 .
$$


From (23) and (22), it follows that $\left\{t\left\|U^{\prime}(t)\right\|_{X}\right\}$ remains bounded as $t \downarrow 0$, so that $[p(t)] \in \mathscr{H}$. This proves the first part of Theorem 4 .

The second part is proved in two steps. First, a function-theoretic argument is used to obtain (21) for $z$ on the positive real axis. Next, the representation (7) is used to extend the estimate to the right half-plane. Fix any $\beta>0$. Since $\left[Z_{p}(t)\right] \in H(L),\left[e^{-\beta t} Z_{p}(t)\right]$ can be continued analytically in $t$, in a sector $\Sigma_{t} \equiv\{\operatorname{Re} t>0,|\operatorname{Arg}(t)| \leq \omega / 2<\pi / 2\}$, with $e^{-\beta t}\left\|Z_{p}(t)\right\|_{L}$ bounded in $\Sigma_{t}$. In fact, with $C_{\beta}$ the constant in (19)

$$
\begin{aligned}
\left\|\left(e^{-\beta t} Z_{p}(t)\right)^{(n)}\right\|_{L} & \leq\left\|\left(e^{-\beta(t / n)} Z_{p}(t / n)\right)^{\prime}\right\|_{L}^{n} \leq\left(n t^{-1} C_{\beta}\right)^{n} \\
& \leq n !\left(e t^{-1} C_{\beta}\right)^{n}, \quad t>0, n \geq 1 .
\end{aligned}
$$

Fix $\omega$ with $0<\omega<2 \tan ^{-1}\left\{1 /\left(e C_{\beta}\right)\right\}$. Then, for $\operatorname{Re} t>0,|\operatorname{Arg}(t)| \leq(\omega / 2)$, the Taylor series

$$
e^{-\beta t} Z_{p}(t)=e^{-\beta \operatorname{Re} t} Z_{p}(\operatorname{Re} t)+\sum_{n=1}^{\infty}(n !)^{-1}(t-\operatorname{Re} t)^{n}\left(e^{-\beta \operatorname{Re} t} Z_{p}(\operatorname{Re} t)\right)^{(n)}
$$

converges uniformly in $B(L)$. Using the isometric isomorphism of $S$ into $B(L)$, it follows that $p(t)$ is holomorphic $\in S$ for $t \in \Sigma_{t}$, and

$$
\left\|e^{-\beta t} p(t)\right\|_{S} \leq\left(1-e C_{\beta}(\operatorname{Re} t)^{-1}|t-\operatorname{Re} t|\right)^{-1} \leq K_{\beta}<\infty, \quad t \in \Sigma_{t} .
$$

Taking the Laplace transform of $p(t)$, we get

$$
\left|e^{-t(\beta+\psi(z))}\right| \leq\left\|e^{-\beta t} p(t)\right\|_{S} \leq K_{\beta}, \quad \operatorname{Re} z \geq 0, t \in \Sigma_{t} .
$$

Let $\xi(z)=\beta+\psi(z)$. It follows from (27) that $\xi$ maps the half-plane $\Pi \equiv$ $\operatorname{Re} z>0$, into the sector $\left\{\Sigma_{z} \equiv|\operatorname{Arg}(z)| \leq(\pi-\omega) / 2\right\}$. From Theorem 2, $\psi(z)$ is holomorphic in $\Pi$ with $\psi(1) \geq 0$. Hence

$$
f(z) \equiv z^{\omega / \pi} \xi(z) / \xi(1),
$$

maps $\Pi$ conformally into itself with $f(1)=1$. Put

$$
z=\frac{1+w}{1-w}, \quad h(w)=f\left(\frac{1+w}{1-w}\right), \quad g(w)=\frac{h(w)-1}{h(w)+1} .
$$

Then, $h(w)$ maps the unit disc into $\Pi$ with $h(0)=1$, and $g(w)$ maps the unit disc into itself with $g(0)=0$. From the Schwarz Lemma applied to $g(w)$, we get

$$
|f(z)|=|h(w)| \leq \frac{1+|w|}{1-|w|}=\frac{|z+1|+|z-1|}{|z+1|-|z-1|} .
$$

Hence, for real $x \geq 1,0<f(x) \leq x$. Therefore, from (28)

$$
0 \leq \psi(x) \leq A x^{\gamma}, \quad x \geq 1 ; \quad A=\psi(1)+\beta, \quad \gamma=(\pi-\omega) / \pi .
$$

We now use the representation (7) to obtain a similar estimate valid in the half-plane $\operatorname{Re} z \geq 0$. The following elementary estimates will be needed:

$$
\left|1-e^{-z}\right| \leq \operatorname{Min}\{|z|, 2\}, \quad \operatorname{Re} z \geq 0 ;
$$




$$
1-e^{-x} \geq \sigma, \quad x \geq 1, \quad 1-e^{-x} \geq \sigma x, \quad 0 \leq x \leq 1,
$$

where $\sigma=1-e^{-1}$. From (7) and (31), we have for $x \geq 1$,

$$
\begin{aligned}
A x^{\gamma} & \geq\left(\int_{0}^{1 / x}+\int_{1 / x}^{\infty}\right)\left(1-e^{-u x}\right) u^{-1} \rho(d u) \\
& \geq \sigma x \int_{0}^{1 / x} \rho(d u)+\sigma \int_{1 / x}^{\infty} u^{-1} \rho(d u) .
\end{aligned}
$$

Therefore, with $\varepsilon=1 / x \leq 1$,

$$
\int_{0}^{\varepsilon} \rho(d u) \leq \sigma^{-1} A \varepsilon^{1-\gamma}, \quad \int_{\varepsilon}^{\infty} u^{-1} \rho(d u) \leq \sigma^{-1} A \varepsilon^{-\gamma} .
$$

If $\operatorname{Re} z \geq 0$, we obtain using (32)

$$
\begin{aligned}
|\psi(z)| & \leq|z| \int_{0}^{\varepsilon} \rho(d u)+2 \int_{\varepsilon}^{\infty} u^{-1} \rho(d u) \\
& \leq \sigma^{-1} A\left(|z| \varepsilon^{1-\gamma}+2 \varepsilon^{-\gamma}\right)
\end{aligned}
$$

if $\varepsilon \leq 1$, on using (35). Setting $\varepsilon=1 /|z|,|z| \geq 1$, we get

$$
|\psi(z)| \leq 3 \sigma^{-1} A|z|^{\gamma}, \quad|z| \geq 1, \quad \operatorname{Re} z \geq 0 .
$$

This concludes the proof of Theorem 4 .

Remark. The restriction $|z| \geq 1$ in (21) is natural: if $\psi(z)=z^{1 / 3}$ for example, the estimate $|\psi(z)| \leq K|z|^{1 / 2}$ is not valid as $z \rightarrow 0$.

Theorem 5. Let $[p(t)] \in \mathscr{I}$. The following statements are equivalent:

(a) $[p(t)] \in \mathscr{G}$.

(b) $\left[Z_{p}(t)\right] \in G(L)$.

(c) $p(t)$ is continuously differentiable $\in S$ for $t>0$, with $\left\|p^{\prime}(t)\right\|_{S}=O(1)$ as $t \downarrow 0$.

(d) $\psi(z)$ is bounded on $\operatorname{Re} z \geq 0$.

(e) $\psi(x)$ is bounded on $x \geq 0$.

(f) $[p(t)]$ is a Compound Poisson family.

Proof. $(\mathrm{a}) \Rightarrow(\mathrm{b}) \Rightarrow(\mathrm{c})$. The argument is the same as that in the first part of Theorem 4, using $\left\|Z_{p}^{\prime}(t)\right\|_{L}=O(1)$ as $t \downarrow 0$.

(c) $\Rightarrow(\mathrm{d}) \Rightarrow(\mathrm{e})$. For sufficiently small $t>0$, we have, on differentiating under the integral sign in $e^{-t \psi(z)}=\mathscr{L}\{p(t)\}$,

$$
\left|\psi(z) e^{-t \psi(z)}\right| \leq\left\|p^{\prime}(t)\right\|_{S}<K<\infty, \quad \operatorname{Re} z \geq 0 .
$$

Hence, $|\psi(z)|<K<\infty, \operatorname{Re} z \geq 0$.

(e) $\Rightarrow(\mathrm{f})$. Let $0 \leq \psi(x)<K$ on $x \geq 0$. Since $[p(t)] \in \mathscr{I}$, we know from Theorem 2 that $\psi(0)=0$ and $\psi^{\prime}(x)$ is completely monotone for $x>0$. Define

$$
Q(z)=1-\psi(z) / K, \quad \operatorname{Re} z \geq 0 .
$$


Then, $Q(0)=1$, and $Q(x)$ is completely monotone for $x>0$. It follows from Bernstein's theorem, (Feller, [5, p. 439]), that $Q(z)$ is the Laplace transform of some probability measure $q$ on $\mathbf{R}^{+}$. Since $\psi(z)=K(1-Q(z)), K>0$, $p(t)$ has the form (13).

(f) $\Rightarrow($ a). Let $p(t)$ have the form (13) and let $U(t)=\langle p(t), T\rangle$ for given $[T(t)] \in C_{0}(X)$. Using the continuous homomorphism $q \mapsto\langle q, T\rangle$ of $S$ into $B(X)$, we get

$$
U(t)=e^{-c t} \sum_{n=0}^{\infty} \frac{(c t)^{n}}{n !}\langle q, T\rangle^{n}, \quad t>0 .
$$

Thus, $[U(t)]$ has the bounded operator $c\{\langle q, T\rangle-I\}$ as its infinitesimal generator, and $[p(t)] \in \mathscr{G}$. This concludes the proof of Theorem 5 .

Theorem 6. If $[p(t)],[q(t)] \in \mathscr{I}[$ resp. $\mathscr{H}, \mathscr{G}]$, then $[p(t) * q(t)] \in \mathscr{I}$ [resp. $\mathscr{H}, \mathscr{G}$ ].

Proof. That $\mathscr{I}$ is closed under convolution is immediate from Theorem 2. Using $(p(t) * q(t))^{\prime}=p^{\prime}(t) * q(t)+p(t) * q^{\prime}(t)$, together with statement (c) in Theorem 4 [resp. Theorem 5], it follows that $\mathscr{H}$ [resp. $\mathscr{G}$ ] is closed under convolution.

\section{Applications}

Example 1. Gamma families $\in \mathscr{H}$.

With fixed $b>0$, let

$$
p_{u}(t)=\frac{b^{t} u^{t-1} e^{-b u}}{\Gamma(t)}, \quad t>0 .
$$

Then

$$
(\partial / \partial t) p_{u}(t)=\left\{\log b+\log u-\frac{\Gamma^{\prime}(t)}{\Gamma(t)}\right\} p_{u}(t), \quad t>0 .
$$

For $0<u<1$, write

$$
(\log u) p_{u}(t)=\left\{2 b^{t / 2} \Gamma(1+t / 2) u^{t / 2}(\log u) p_{u}(t / 2)\right\}\{\Gamma(1+t)\}^{-1},
$$

and for $u \geq 1$, write

$$
(\log u) p_{u}(t)=\left\{2^{t}\left(e^{-b u / 2} \log u\right)(b / 2)^{t} u^{t-1} e^{-b u / 2}\right\}\{\Gamma(t)\}^{-1} .
$$

Let

$$
K_{1}=\max _{0 \leq v \leq 1}\{v|\log v|\}, \quad K_{2}=\sup _{u \geq 1}\left\{e^{-b u / 2} \log u\right\} .
$$

From (42)-(45), we have

$$
\left\|p^{\prime}(t)\right\|_{S} \leq 2^{t} K_{2}+|\log b|+\frac{\left|\Gamma^{\prime}(t)\right|}{\Gamma(t)}+\frac{4 K_{1} b^{t / 2} \Gamma(1+t / 2)}{t \Gamma(1+t)}, \quad t>0 .
$$


The only singularity in $\Gamma^{\prime}(t) / \Gamma(t), t \geq 0$, is a simple pole at $t=0$; see Olver, [8, p. 39]. Also, $p^{\prime \prime}(t)=p^{\prime}(t / 2) * p^{\prime}(t / 2)$. Thus, $p(t)$ is continuously differentiable $\in S$ for $t>0$, and $\left\{t\left\|p^{\prime}(t)\right\|_{S}\right\}$ remains bounded as $t \downarrow 0$. By Theorem 4, $[p(t)] \in \mathscr{H}$.

Example 2 (Corollary). If $[T(t)]=\left[e^{-t A}\right] \in C_{0}(X)$, then $-\log (A+I)$, where

$$
\{\log (A+I)\} x=\int_{1}^{\infty} s^{-1}(A+s I)^{-1} A x d s, \quad x \in D(A),
$$

is the infinitesimal generator of $[S(t)]=\left[(A+I)^{-t}\right] \in H(X)$.

Let $[p(t)] \in \mathscr{I}$ have the exponent $\psi(z)$, and let $\rho$ be the measure on $\mathbf{R}^{+}$in (7). Let $U(t)=\langle p(t), T\rangle$. A formula for the generator of $[U(t)]$ is known, which generalizes Theorem 2 and the representation (7); see Phillips, [10], Nelson, [7], and Feller, [5, p. 458]. We have

$$
\begin{aligned}
{[U(t)] } & =\left[e^{-t \psi(A)}\right], \\
\psi(A) x & =\int_{\mathbf{R}^{+}} u^{-1}\left(I-e^{-u A}\right) x \rho(d u), \quad x \in D(A) .
\end{aligned}
$$

In fact, $\psi(A)$ is the closure of its restriction to $D(A)$. Choosing $[p(t)]$ to be the Gamma family (41) with $b=1$, we have $\psi(z)=\log (1+z), \rho(d u)=e^{-u} d u$, and

$$
\begin{aligned}
\{\log (A+I)\} x & =\int_{\mathbf{R}^{+}}\left(I-e^{-u A}\right) x\left\{\int_{1}^{\infty} e^{-u s} d s\right\} d u \\
& =\int_{1}^{\infty} s^{-1}(A+s I)^{-1} A x d s, \quad x \in D(A) .
\end{aligned}
$$

The result follows on viewing $[S(t)]$ as being subordinated to $[T(t)]$ through the Gamma family.

The Hausdorff-Young theorem on Fourier transforms may be combined with Theorem 4 to obtain another proof of the fact that the one-sided Lévy stable families $\in \mathscr{H}$ :

Example 3. Fix $\alpha$ with $0<\alpha<1$, and let $\left[p^{\alpha}(t)\right]$ have the exponent $\psi(z)=$ $z^{\alpha}$. Then, $\left[p^{\alpha}(t)\right] \in \mathscr{H}$.

From Theorem $2,\left[p^{\alpha}(t)\right] \in \mathscr{J}$ for $0<\alpha<1$. We verify statement (c) of Theorem 4 for $(d / d t) p^{\alpha}(t)$. Let $b=\cos (\alpha \pi / 2)>0$, and let $C$ be a generic positive constant. Let $q_{t, \alpha}(u) \equiv(\partial / \partial t) p_{u}^{\alpha}(t), u \geq 0$. For real $y, Q_{t, \alpha}(y) \equiv$ $-(i y)^{\alpha} e^{-t(i y)^{\alpha}}$ and $(\partial / \partial y) Q_{t, \alpha}(y)$ are, respectively, the Fourier transforms of the densities $q_{t, \alpha}(u)$ and $g_{t, \alpha}(u) \equiv i u q_{t, \alpha}(u)$. Moreover

$$
\begin{gathered}
\left|Q_{t, \alpha}(y)\right|=|y|^{\alpha} e^{-b t|y|^{\alpha}}, \\
\left|(\partial / \partial y) Q_{t, \alpha}(y)\right| \leq \alpha|y|^{\alpha-1}\left(1+t|y|^{\alpha}\right) e^{-b t|y|^{\alpha}} .
\end{gathered}
$$


Let $1<r<\min \left\{2,(1-\alpha)^{-1}\right\}$, let $s=r /(r-1)$, and let \|\|$_{r}$ denote the $L^{r}(-\infty, \infty)$ norm. The change of variables $v=t y^{\alpha}$ shows that

$$
t\left\|Q_{t, \alpha}\right\|_{r} \leq C t^{-1 / \alpha r}, \quad t\left\|(\partial / \partial y) Q_{t, \alpha}\right\|_{r} \leq C t^{1 / \alpha s} .
$$

Using the Hausdorff-Young inequality, (Rudin, [11, p. 247]), we obtain

$$
t\left\|q_{t, \alpha}\right\|_{s} \leq C t^{-1 / \alpha r}, \quad t\left\|g_{t, \alpha}\right\|_{s} \leq C t^{1 / \alpha s} .
$$

Next, for any $v>0$, Hölder's inequality gives

$$
\begin{gathered}
\int_{0}^{v}\left|q_{t, \alpha}(u)\right| d u \leq\left\|q_{t, \alpha}\right\|_{s} v^{1 / r}, \\
\int_{v}^{\infty} u\left|q_{t, \alpha}(u)\right|(1 / u) d u \leq\left\|g_{t, \alpha}\right\|_{s} v^{-1 / s} .
\end{gathered}
$$

Therefore, on choosing $v=t^{1 / \alpha}$, it follows from (53), (54), and (55), that $q_{t, \alpha}(u) \in L^{1}\left(\mathbf{R}^{+}\right)$for each $t>0$, and $\left\|q_{t, \alpha}\right\|_{1} \equiv\left\|(d / d t) p^{\alpha}(t)\right\|_{S}=O\left(t^{-1}\right)$ as $t \downarrow 0$. Hence, $\left[p^{\alpha}(t)\right] \in \mathscr{H}$.

Evidently, a large number of objects $\in \mathscr{H}$ can be created by convolutions. Further objects $\in \mathscr{H}$ may be generated by means of the following construction:

Example 4. Subordination of convolution semigroups.

Let $\left[p_{1}(t)\right] \in \mathscr{H},\left[p_{2}(t)\right] \in \mathscr{I}$, and consider the convolution semigroup $\left[Z_{p_{2}}(t)\right] \in C_{0}(L)$. Let $U(t)=\left\langle p_{1}(t), Z_{p_{2}}\right\rangle$. Then, $[U(t)] \in H(L)$ is the convolution semigroup $\left[Z_{p_{3}}(t)\right]$, where

$$
p_{3}(t)=\int_{\mathbf{R}^{+}} p_{2}(u) p_{1}(t)(d u), \quad t>0 .
$$

Using Laplace transforms, it is easily seen that if $\psi_{j}(z)$ is the exponent $\left[p_{j}(t)\right]$, $j=1,2$, then $\left[p_{3}(t)\right]$ has the exponent $\psi_{3}(z)=\psi_{1}\left(\psi_{2}(z)\right)$. Since $\psi_{3}(x)$ vanishes at 0 and has a completely monotone derivative on $x>0$, it follows from Theorem 2 that $\left[p_{3}(t)\right] \in \mathscr{I}$. Statement $(\mathrm{b})$ of Theorem 4 shows that $\left[p_{3}(t)\right] \in \mathscr{H}$.

We now construct two distinct classes of objects $\in \mathscr{I} \backslash \mathscr{H}$.

Example 5. If $[p(t)] \in \mathscr{I}$, then $[q(t)]=\left[p(t) * \delta_{t}\right] \in \mathscr{I} \backslash \mathscr{H}$.

If $[p(t)]$ has the exponent $\psi(z),[q(t)]$ has the exponent $z+\psi(z)$. From (21) in Theorem 4, $[q(t)] \notin \mathscr{H}$.

Example 6. Let $\left\{\beta_{n}\right\}_{n=0}^{\infty}$ and $\left\{a_{n}\right\}_{n=0}^{\infty}$ be any two sequences satisfying

$$
0<\beta_{n}<1 ; \quad 0<a_{n} ; \quad \lim _{n \rightarrow \infty} \beta_{n}=1 ; \quad \sum_{n=0}^{\infty} a_{n}<\infty ;
$$

and define

$$
\psi(z)=\sum_{n=0}^{\infty} a_{n} z^{\beta_{n}}, \quad \operatorname{Re} z>0 .
$$


Then, $\psi(z)$ is the exponent of some $[p(t)] \in \mathscr{J} \backslash \mathscr{H}$. The same is true for $\psi(\varphi(z))$, whenever $\varphi(z)$ is a function of the form (7) that does not satisfy (21).

The infinite series of holomorphic functions (58) converges uniformly on compact subsets of the half-plane $\operatorname{Re} z>0$, to a holomorphic $\psi(z)$ with $\psi(0)=0$. In particular, termwise differentiation is permissible. It follows that $\psi(x)$ has a completely monotone derivative on $x>0$. By Theorem 2, $[p(t)] \in \mathscr{F}$. Since each $a_{n}>0, \psi(z)$ cannot satisfy $(21)$ on $x>0$, and $[p(t)] \notin \mathscr{H}$. Similarly, $\psi(\varphi(z))$ is the exponent of some $[r(t)] \in \mathscr{I}$, since it vanishes at zero, and has a completely monotone derivative for $x>0$. That $[r(t)] \notin \mathscr{H}$ may be seen by examining the series:

$$
\begin{aligned}
\psi(\varphi(x)) & =\sum_{n=0}^{\infty} a_{n}\{\varphi(x)\}^{\beta_{n}}, \quad x>0, \\
& >a_{m}\{\varphi(x)\}^{\beta_{m}}, \quad m>0 .
\end{aligned}
$$

Fix any $\gamma$ with $0<\gamma<1$, fix $m>0$ such that $\gamma<\beta_{m}<1$, and let $\alpha_{m}=\left(\gamma / \beta_{m}\right)<1$. Then, $x^{-\gamma} \psi(\varphi(x))>a_{m}\left\{x^{-\alpha_{m}} \varphi(x)\right\}^{\beta_{m}}$, and cannot remain bounded as $x \rightarrow \infty$.

\section{REFERENCES}

1. A. V. Balakrishnan, An operational calculus for infinitesimal generators of semigroups, Trans. Amer. Math. Soc. 91 (1959), 330-353.

2. __ Fractional powers of closed operators and the semigroups generated by them, Pacific J. Math. 10 (1960), 419-437.

3. S. Bochner, Diffusion equation and stochastic processes, Proc. Nat. Acad. Sci. U.S.A. 35 (1949), 368-370.

4. $\ldots$, Harmonic analysis and the theory of probability, Univ. of California Press, Berkeley, Calif., 1955.

5. W. Feller, An introduction to probability theory and its applications, vol. 2, 2nd ed., Wiley, New York, 1971.

6. E. Hille and R. S. Phillips, Functional analysis and semigroups, Amer. Math. Soc. Colloq. Publ., vol. 31, Providence, R.I., 1957.

7. E. Nelson, A functional calculus using singular Laplace integrals, Trans. Amer. Math. Soc. 88 (1958), 400-413.

8. F. W. J. Olver, Asymptotics and special functions, Academic Press, New York, 1974.

9. A. Pazy, Semigroups of linear operators and applications to partial differential equations, Springer-Verlag, New York, 1983.

10. R. S. Phillips, On the generation of semigroups of linear operators, Pacific J. Math. 2 (1952), 343-369.

11. W. Rudin, Real and complex analysis, McGraw-Hill, New York, 1966.

12. K. Yosida, An abstract analyticity in time for solutions of a diffusion equation, Proc. Japan Acad. 35 (1959), 109-113.

13. _ Fractional powers of infinitesimal generators and the analyticity of the semigroups generated by them, Proc. Japan Acad. 36 (1960), 86-89. 
14. _ On a class of infinitesimal generators and the integration problem of evolution equations, Proc. Fourth Berkeley Sympos. Math. Stat. Probab. Volume 2, Contributions to Probability Theory, (Jerzy Neyman, Ed.), Univ. of California Press, Berkeley, Calif., 1961, pp. 623-633.

15. __ On holomorphic Markov processes, Proc. Japan Acad. 42 (1966), 313-317.

Center for Computing and Applied Mathematics, National Institute of Standards AND TeChNology, Gaithersburg, Maryland 20899

Department of Mathematics, University of California at Berkeley, Berkeley, CaliFORNIA 94720 